\title{
A Rare Case of Male Bullous Lupus Erythematosus Complicated with Subsequent Annular Hypopigmentation
}

\author{
Saori Itoi ${ }^{a} \quad$ Atsushi Tanemura $^{a} \quad$ Chie Tsuji $^{a} \quad$ Shun Kitaba ${ }^{a}$ \\ Akinori Yokomi $^{\mathrm{a}} \quad$ Ichiro Katayama $^{\mathrm{a}} \quad$ Chiharu Tateishi $^{\mathrm{b}}$ Daisuke Tsuruta ${ }^{\mathrm{b}}$ \\ aDepartment of Dermatology, Osaka University Graduate School of Medicine, Suita, and \\ ${ }^{b}$ Department of Dermatology, Osaka City University Graduate School of Medicine, Osaka, \\ Japan
}

\section{Key Words}

Bullous lupus erythematosus · Annular hypopigmentation · Ultrastructural analysis

\begin{abstract}
A 57-year-old male had been suffering from an itchy map-shaped symmetrical erosive erythema with a crust that was attached to his upper arm and buttock, and occasionally he suffered from spiking fever. Laboratory examinations showed neither anti-desmoglein $1 / 3$ antibodies nor anti-BP 180 antibodies, and he fulfilled the criteria for a diagnosis of systemic lupus erythematosus (SLE). Histologically, there was eosinophilic necrosis of keratinocytes, liquefaction and degradation with severe lymphocyte infiltration into the epidermis and subepidermal blister formation, suggestive of a variant of SLE, bullous lupus erythematosus (BLE). One month after remission of BLE, peculiar annular hypopigmentation appeared on the peripheral borders. An immunohistochemical analysis showed a decrease in Melan A-positive melanocytes and concomitant pigment incontinentia, with dense infiltration of $\mathrm{CD} 8^{+} \mathrm{T}$ cells and IL-17A ${ }^{+}$Th17 cells. An ultrastructural analysis revealed a decrease, but not a complete disappearance, of both melanocytes and melanosomes, and no impairment in melanosomal transfer. In this case report, we would like to introduce the development of annular depigmentation complicated with BLE, and discuss the effects of lupus condition on melanocyte damage based on immunohistological and electromicroscopic findings of those vitiliginous lesions.


Itoi et al:: A Rare Case of Male Bullous Lupus Erythematosus Complicated with Subsequent Annular Hypopigmentation

\section{Introduction}

Bullous lupus erythematosus (BLE) is a rare subset of systemic lupus erythematosus (SLE) [1]. We experienced a rare case of BLE complicated with annular hypopigmentation. The bullous erythema and subsequent annular hypopigmentation responded to systemic steroid treatment. There have been few studies addressing hypopigmented lesions in lupus erythematosus (LE). In this study, we would like to introduce a rare case of SLE, and show immunohistochemical and ultrastructural findings of the vitiliginous lesions following erosive erythema.

\section{Case Report}

A 57-year-old male patient had noticed itchy erythema on the frontal chest in May 2011, and thereafter experienced disease enlargement and partial crust formation. Since no clinical effect of $10 \mathrm{mg} /$ day of oral prednisolone was observed, he was referred to our hospital on July 7, 2011. In terms of the clinical findings at the first visit, symmetric red papules and centrifugal erythema, surrounded by an annular erosive crust, were distributed on the upper extremities and trunk in line with the seborrheic region. The center of the erythematous lesions in the skin tended to be notably resolved (fig. 1a), while the mucosal areas were intact. A fever and symmetrical bullous erythema worsened 1 week after admission (fig. 1b). Although his skin disease was initially suspected to be a bullous disease, neither anti-desmoglein 1/3 nor anti-BP 180 antibodies were detected.

Laboratory tests revealed cytopenia (WBC, 10,470/ $\mu \mathrm{l}$; Neu, 72.6\%; lymph, 13.0\%; mono, 14.0\%; eosino, 0.1\%; baso, $0.3 \%$; RBC, $3.85 \times 10^{6} / \mu \mathrm{l}$; Hb, $12.7 \mathrm{~g} / \mathrm{dl}$; Ht, 38.5\%; platelet, $180 \times$ $\left.10^{3} / \mu \mathrm{l}\right)$, ANA (1/320, homogeneous + speckled pattern), positive anti-Sm antibodies, antiRNP, anti-cardiolipin antibodies, and low C3 and C4 levels. Metal patch and photo tests were negative, and no internal malignancy was detected by whole-body imaging. A biopsy of the erythematous lesion showed individual apoptotic keratinocytes and liquefaction with partial cleft formation on the epidermis, and lymphocytes predominantly infiltrated in the upper dermis and perivascular area (fig. 1b). Pigment incontinentia was notable, along with severe inflammation (fig. 1c). The direct immunofluorescence findings of the uninvolved lesion were granular deposition of IgG and IgM in the basal membrane.

These laboratory and histological findings led to a final diagnosis of BLE type I. In an immunohistochemical analysis for immunocompetent cells, $\mathrm{CD}^{+}$cells, instead of CD4 ${ }^{+}$cells, and HLA-DR+-activating T cells were found to have densely infiltrated into the epidermis of the erythematous lesion. The number of CD1a+ Langerhans cells was decreased. The CD68 ${ }^{+}$ macrophages densely infiltrated into the upper dermis, and there was a slight infiltration of IL-17A+ cells and Foxp3 $3^{+}$cells (fig. $2 \mathrm{a}-\mathrm{h}$ ).

An increased dose of oral prednisolone was effective not only for spiking fever, cytopenia and hypocomplement level, but also crusted bullae and fused erythema (fig. 3a). Instead, annular incomplete hypopigmentation tracing the crusted lesions was noted 1 month later (fig. 3b). Our histological observations of the hypopigmented lesion showed a decrease, but not total absence, of melanosomes and melanocytes detected by Masson-Fontana (fig. 3d-f) and Melan-A (fig. 3g-i) staining, respectively. In addition, an ultrastructural analysis of a hypopigmented lesion revealed attenuated melanin production, with the presence of immature melanosomes in the remaining melanocytes, whereas no disturbance of melanin transfer to adjacent keratinocytes was noted (fig. 3j, k). The hypopigmented lesions recovered 2 weeks later, without any additional treatment (fig. 3c). 
Itoi et al:: A Rare Case of Male Bullous Lupus Erythematosus Complicated with Subsequent Annular Hypopigmentation

\section{Discussion}

We experienced a rare case of BLE complicated with annular hypopigmentation. The bullous erythema and subsequent annular hypopigmentation responded to systemic steroid treatment. BLE is a rare variant of SLE, with an incidence of less than 0.2 cases per million per year, and represents only $2-3 \%$ of the cases of autoimmune subepidermal bullous dermatoses [2]. Clinically, BLE is characterized by a disseminated vesiculobullous skin rash that may or may not be limited to areas exposed to light. Although it is considered to be an acquired bullous disease caused by anti-type VII collagen antibodies, the same as epidermolysis bullosa acquisita [2], the bullous lesions in BLE usually remit without scar formation, which is common in cases of epidermolysis bullosa acquisita. Since antibodies such as antidesmoglein 1/3 antibodies and anti-BP 180 antibodies were not detected, the present case of BLE was diagnosed according to the diagnostic criteria [3].

A histopathological examination revealed subepidermal blisters with neutrophilic microabscesses in the dermal papillae, perivascular inflammatory infiltration composed of lymphomononuclear cells, and in some cases, leukocytoclastic vasculitis [4]. Our case also showed individual apoptotic keratinocytes, liquefaction with a partial cleft on the epidermis and lymphocytes predominantly infiltrating into the upper dermis and perivascular area.

In our case, the annular hypopigmented macules appeared along the edge of the erythematous lesion. Franca and de Souza [5] reviewed the histological features of 12 patients with depigmented or hypopigmented lesions in 220 patients with cutaneous LE, and the most common histological findings were summarized as: cellular infiltration (75\%), hyperkeratosis (66.7\%), thinning and flattening of the epidermis (58.3\%) and lower number of melanocytes compared to normal skin. We observed similar histological findings in our case, including apoptotic keratinocytes, atrophy of the epidermis and superficial lymphocytic infiltration in the dermis and a decreased number of melanocytes.

For a more detailed observation, we carried out the immunohistochemical and ultrastructural analyses for both the erythematous and subsequent hypopigmented lesions. For the immunohistochemical analysis, $\mathrm{CD}^{+}$cells (instead of $\mathrm{CD} 4^{+}$cells) and HLA-DR+-activating $\mathrm{T}$ cells were found to have densely infiltrated into the epidermis of the erythematous lesion. There was a decrease in the number of $\mathrm{CD} 1 \mathrm{a}^{+}$Langerhans cells. While the $\mathrm{CD}^{+} 8^{+}$macrophages densely infiltrated the upper dermis, there was a slight infiltration of $\mathrm{IL}^{-17 \mathrm{~A}^{+} \text {cells }}$ and Foxp $3^{+}$cells. Liquefaction, degeneration and dense infiltration of CD8 ${ }^{+} \mathrm{T}$ cells into the epidermis were detected, possibly resulting in hypopigmentation accompanied by LE. Whereas the CD8 ${ }^{+} \mathrm{T}$ cells were densely found in the affected lesions, the IL- $17 \mathrm{~A}^{+}$cells and Foxp $3^{+}$regulatory $\mathrm{T}$ cells were sparsely present. Moreover, we detected a large number of Th17 cells infiltrating in the depigmented lesions compared to the uninvolved skin in nonsegmental vitiligo patients $[6,7]$.

With regard to ultrastructural findings, while melanosome production in melanocytes was attenuated, adjacent keratinocytes took over a significant number of mature melanosomes, indicating that there were decreases in both the melanocyte and melanosome number without impaired melanosome transfer to keratinocytes. Taking into consideration both these immune responses and the lupus condition, we considered the following process to be involved in the pathogenesis of the annular hypopigmentation; Cytotoxic $\mathrm{T}$ cells penetrated into the epidermis followed by melanocyte destruction. Thereafter, pigment incontinentia followed by the recruitment of numerous $\mathrm{CD}^{+} 8^{+}$melanophages in the upper dermis induced activation of melanin-specific antigen presentation, resulting in positive loop cellular immunity. The CD8 ${ }^{+}$cytotoxic cell-related immune response was thus induced, and melanocyte were attacked, leading to basal membrane degeneration, which may have played 
Itoi et al:: A Rare Case of Male Bullous Lupus Erythematosus Complicated with Subsequent Annular Hypopigmentation

a major role in the hypopigmentation associated with LE. Early induction of systemic prednisolone treatment may have led to lesion improvement owing to the sufficient inhibition of this vicious disease cycle.

\section{Conclusion}

We experienced a case of LE associated with annular hypopigmentation. We suppose that a $\mathrm{CD}^{+}$cytotoxic cell-related immune response in addition to basal membrane degeneration may play a major role in the development of hypopigmentation associated with LE. Early administration of effective treatment may be important for restoration of the pigmentation.

Although annular hypopigmentation is generally regarded as secondary hypopigmentation after local inflammation, annular hypopigmentation following BLE also occurs in some patients. We believe that using a detailed morphological and hematological analysis in additional patient samples will help to elucidate the exact mechanism(s) underlying the development of hypopigmentation in patients with BLE.

\section{Disclosure Statement}

The authors have no conflicts of interest to disclose.

\section{References}

1 Vassileva S: Bullous systemic lupus erythematosus. Clin Dermatol 2004;22:129-138.

12 Tincopa M, Puttgen KB, Sule S, Cohen BA, Gerstenblith MR: Bullous lupus: an unusual initial presentation of systemic lupus erythematosus in an adolescent girl. Pediatr Dermatol 2010;27:373-376.

-3 Camisa C, Sharma HM: Vesiculobullous systemic lupus erythematosus. Report of two cases and a review of the literature. J Am Acad Dermatol 1983;9:924-933.

4 Obermoser G: Lupus erythematosus and the skin: a journey at times perplexing, usually complex, often challenging, and evermore exhilarating. Lupus 2010;19:1009-1011.

5 Franca AF, de Souza EM: Histopathology and immunohistochemistry of depigmented lesions in lupus erythematosus. J Cutan Pathol 2010;37:559-564.

-6 Kotobuki Y, Tanemura A, Yang L, Itoi S, Wataya-Kaneda M, Murota H, Fujimoto M, Serada S, Naka T, Katayama I: Dysregulation of melanocyte function by Th17-related cytokines: significance of Th17 cell infiltration in autoimmune vitiligo vulgaris. Pigment Cell Melanoma Res 2012;25:219-230.

7 Nordlund JJ, Abdel-Malek ZA: Mechanisms for post-inflammatory hyperpigmentation and hypopigmentation. Prog Clin Biol Res 1988;256:219-236. 


\section{Case Reports in Dermatology}
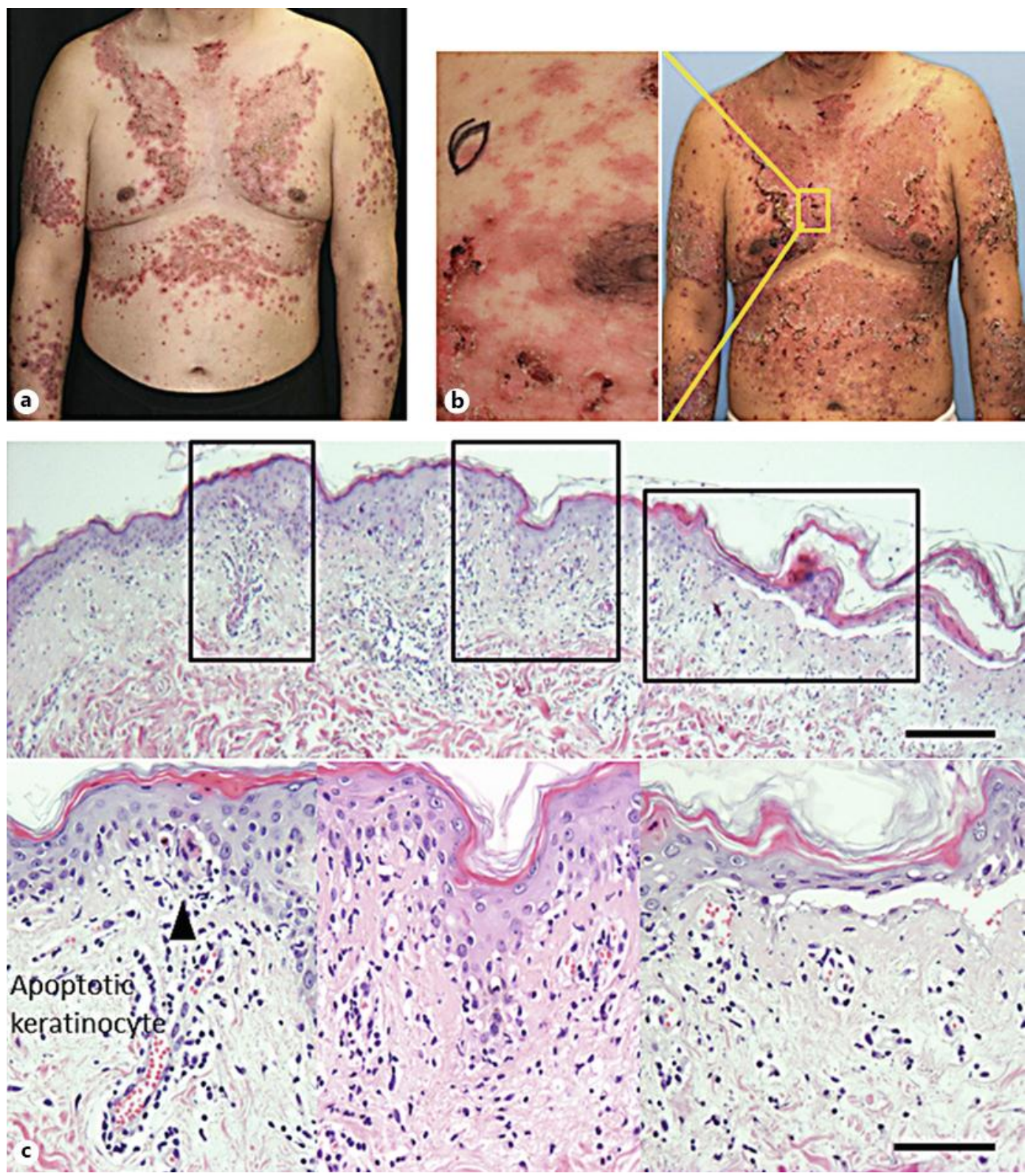

Fig. 1. a Clinical features at the first visit. Symmetrical red papules and centrifugal erythema surrounded by an annular erosive crust were distributed on the upper extremities and trunk in line with the seborrheic region. The center of the erythematous lesions tended to be notably resolved. Mucosal areas, such as the oral cavity and penis, were not involved. b Clinical features 1 week after consultation. Spiking fever and symmetrical bullous erythema worsened 1 week after admission, suggestive of pemphigus foliaceus. c Histopathological features on the erythematous lesion. Bars $=100 \mu \mathrm{m}$. 


\section{Case Reports in Dermatology}

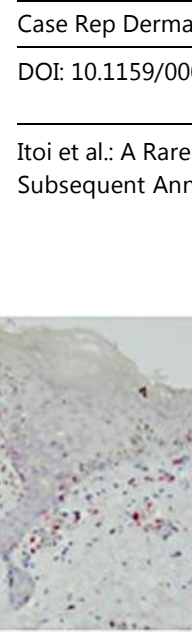

CD1a

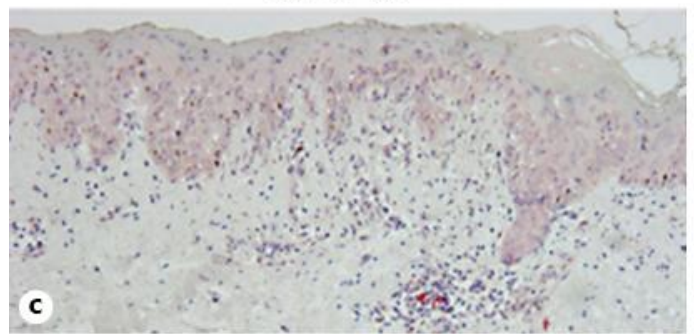

CD68

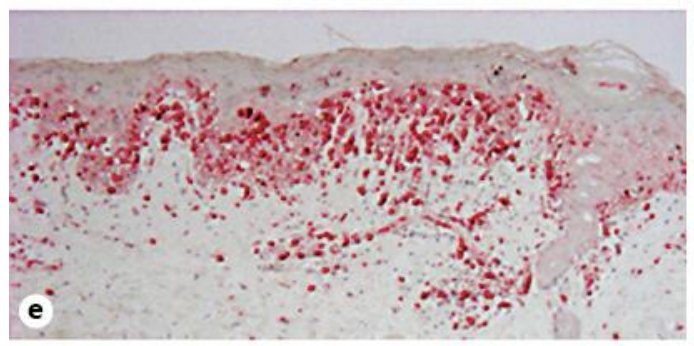

IL-17A

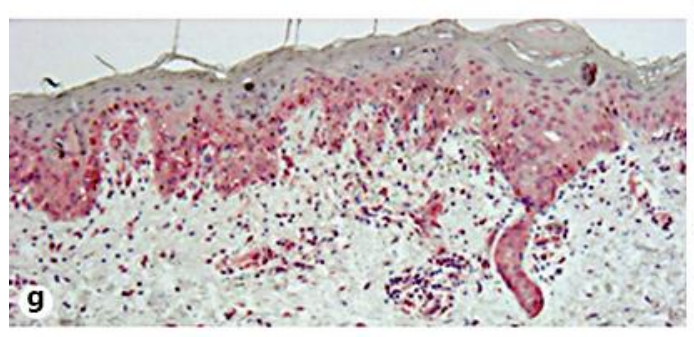

CD8

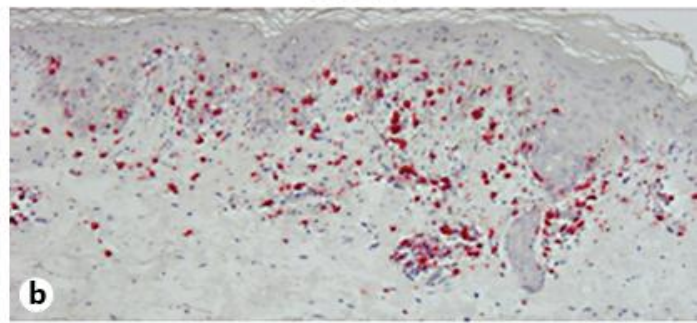

HLA-DR

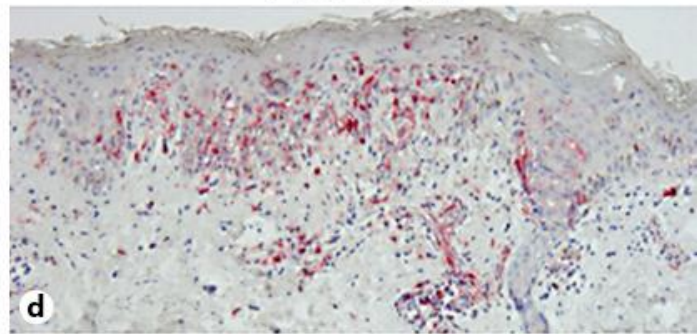

CD163

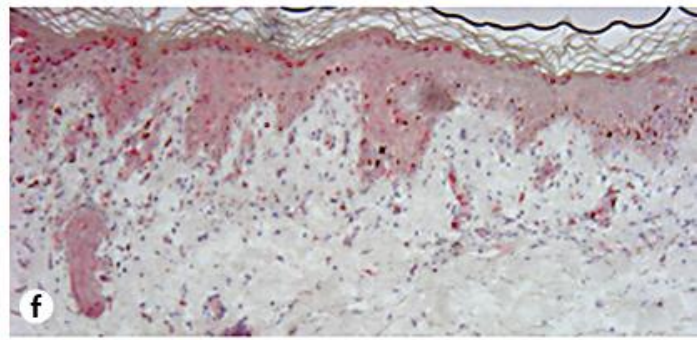

\section{Foxp3}

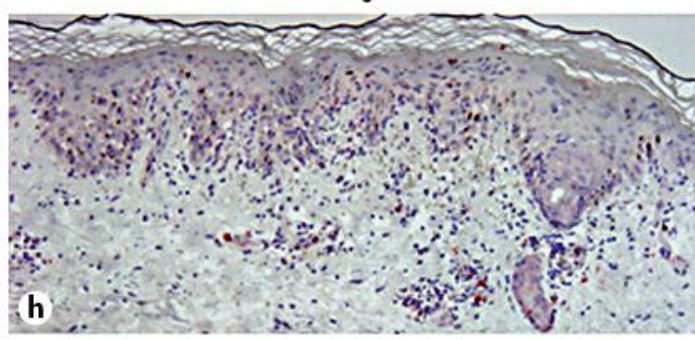

Fig. 2. Results of immunohistochemical analysis for immunocompetent cells. Paraffin-embedded tissue samples from the patient were stained with CD4 (a), CD8 (b), CD1a (c), HLA-DR (d), CD68 (e), CD163 (f), IL-17A (g) and Foxp3 (h). CD8 ${ }^{+}$cells, instead of CD4+ cells, and HLA-DR+-activated T cells densely infiltrated into the epidermis of the erythematous lesion. CD1 $\mathrm{a}^{+}$Langerhans cells were decreased in number. While $\mathrm{CD} 68^{+}$macrophages densely infiltrated into the upper dermis, there was only slight infiltration of IL-17A $\mathrm{A}^{+}$cells and Foxp3 $3^{+}$cells. Bar $=250 \mu \mathrm{m}$. 


\section{Case Reports in Dermatology}
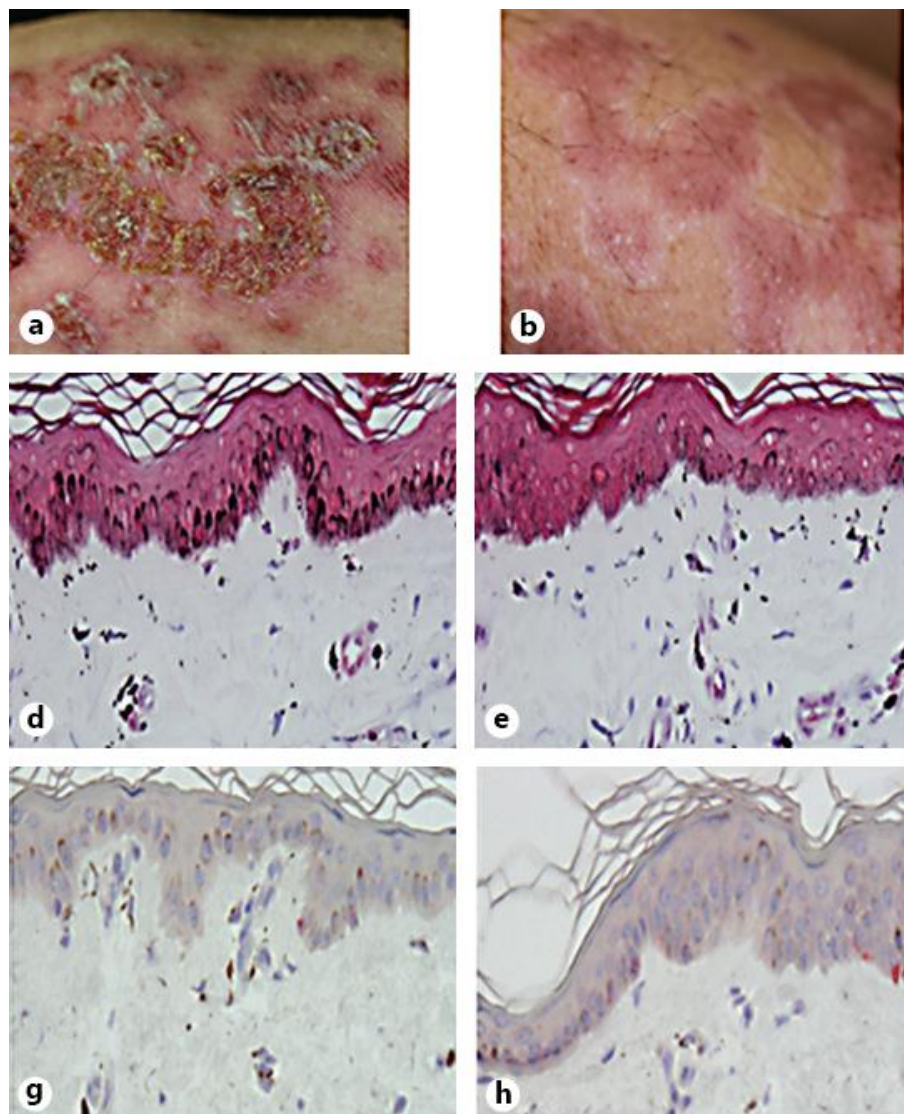

Itoi et al:: A Rare Case of Male Bullous Lupus Erythematosus Complicated with Subsequent Annular Hypopigmentation

\begin{tabular}{l|l}
\hline Case Rep Dermatol 2014;6:91-97 & $\begin{array}{l}\text { C 2014 S. Karger AG, Basel } \\
\text { www.karger.com/cde }\end{array}$ \\
\hline DOI: $10.1159 / 000360980$ &
\end{tabular}

b
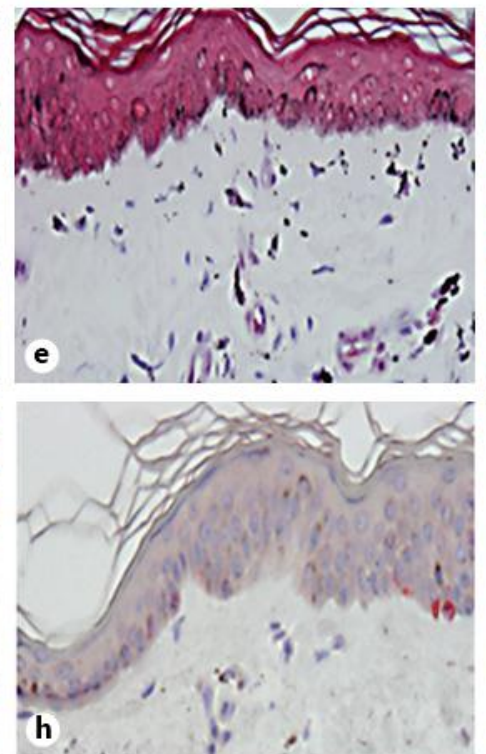
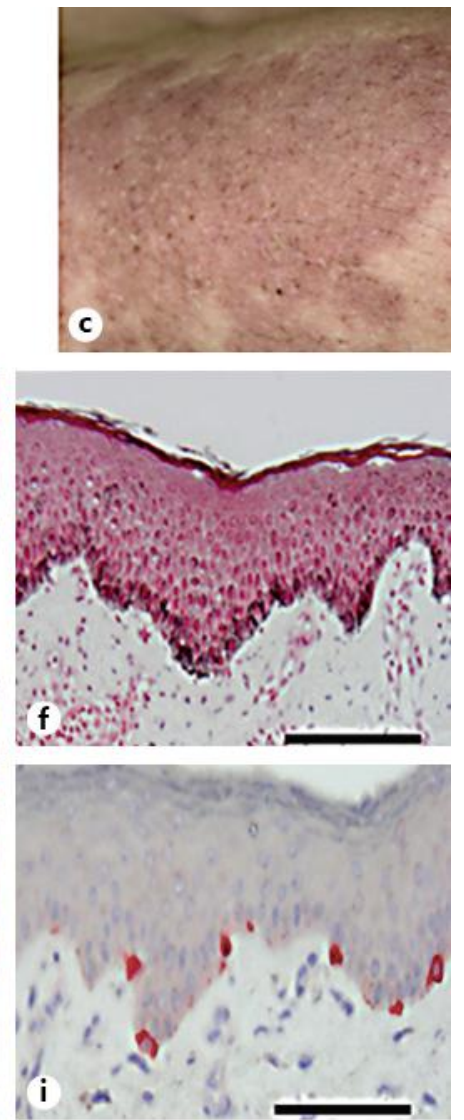
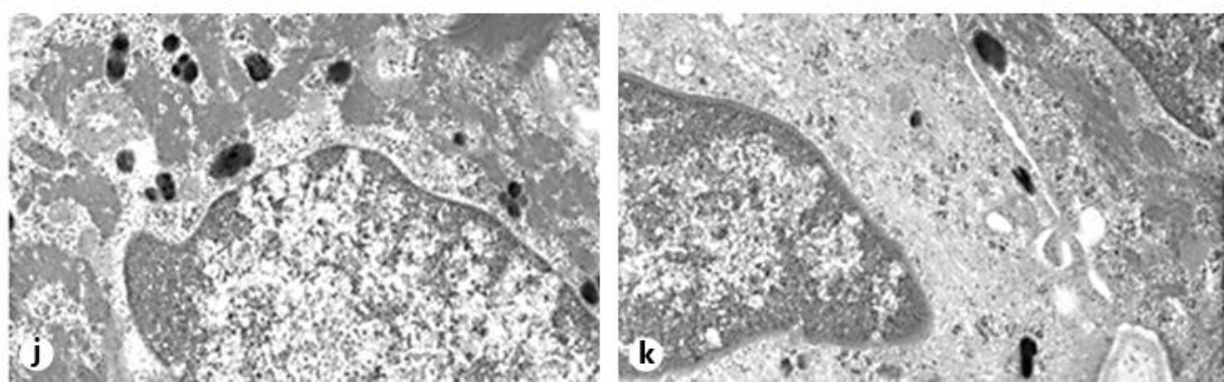

Fig. 3. Clinical course after admission, and the distribution of melanocytes and melanosomes in each lesion. a The image shows symmetrical bullous erythema that worsened 1 week after admission. $\mathbf{b}$ The crusted bulla and fused erythema remitted after systemic steroid treatment. Twenty-eight days after starting the treatment, annular hypopigmentation appeared corresponding to the edge of the erythematous lesion. c The hypopigmentation was decreased 40 days after admission. $\mathbf{d - f}$ Masson-Fontana staining of erythematous (d), annular hypopigmented (e) and uninvolved lesions (f). The erythematous and hypopigmented lesions were accompanied by extensive pigment incontinentia. In contrast, basal melanosomes were increased in the erythematous lesion. Bars $=100 \mu \mathrm{m}$. g-i Melan-A immunohistochemical staining of erythematous ( $\mathbf{g}$ ), hypopigmented (h) and uninvolved lesion (i). The number of melanocytes was decreased in the erythematous and hypopigmented lesions compared to the uninvolved lesion. Bar $=100 \mu \mathrm{m}$. j, k. Results of the ultrastructural analysis for the hypopigmented lesion. While melanosome production in melanocytes was attenuated, adjacent keratinocytes (j) possessed a significant number of mature melanosomes (k), suggesting no trafficking disturbance. Original magnification $\times 15,000$. 